\title{
The dependence of cusp ion signatures on the reconnection rate
}

\author{
S. K. Morley ${ }^{1}$ and M. Lockwood ${ }^{1,2}$ \\ ${ }^{1}$ Department of Physics and Astronomy, University of Southampton, UK \\ ${ }^{2}$ Rutherford Appleton Laboratory, Chilton, Oxfordshire, UK
}

Received: 17 December 2001 - Revised: 30 August 2002 - Accepted: 18 September 2002

\begin{abstract}
The interpretation of structure in cusp ion dispersions is important for helping to understand the temporal and spatial structure of magnetopause reconnection. "Stepped" and "sawtooth" signatures have been shown to be caused by temporal variations in the reconnection rate under the same physical conditions for different satellite trajectories. The present paper shows that even for a single satellite path, a change in the amplitude of any reconnection pulses can alter the observed signature and even turn sawtooth into stepped forms and vice versa. On 20 August 1998, the Defense Meteorological Satellite Program (DMSP) craft F-14 crossed the cusp just to the south of Longyearbyen, returning on the following orbit. The two passes by the DMSP F-14 satellites have very similar trajectories and the open-closed field line boundary (OCB) crossings, as estimated from the SSJ/4 precipitating particle data and Polar UVI images, imply a similarly-shaped polar cap, yet the cusp ion dispersion signatures differ substantially. The cusp crossing at 08:54 UT displays a stepped ion dispersion previously considered to be typical of a meridional pass, whereas the crossing at 10:38 UT is a sawtooth form ion dispersion, previously considered typical of a satellite travelling longitudinally with respect to the OCB. It is shown that this change in dispersed ion signature is likely to be due to a change in the amplitude of the pulses in the reconnection rate, causing the stepped signature. Modelling of the low-energy ion cutoff under different conditions has reproduced the forms of signature observed.
\end{abstract}

Key words. Ionosphere (particle precipitation) Magnetospheric physics (energetic particles, precipitating, magnetopause, cusp and boundary layers)

\section{Introduction}

The origin of cusp ion dispersion signatures has been dealt with for observing satellites over a range of altitudes. In this

Correspondence to: S. Morley (skm@phys.soton.ac.uk) paper we shall be concentrating on low-altitude satellite observations.

\subsection{Cusp ion dispersion signatures}

During sub-solar reconnection under southward IMF $\left(B_{z}<0\right)$, newly-opened field lines allow particles from the magnetosheath to precipitate through the magnetospheric cusps into the ionosphere. The velocity dispersion as the particles travel along field-lines from the point of particle injection means that the more energetic particles of any one species will reach the ionosphere before the less energetic particles. The lowest energy ions measured at any point are, therefore, from the point of reconnection of that field line. This means that the low-energy ion cutoff is directly related to the time elapsed since reconnection. On the boundaries between regions of newly-opened flux, a discontinuity will be observed if the reconnection is pulsed. The form that the cusp ion dispersion will take in low-altitude satellite observations has been modelled by Lockwood and Davis (1996), showing that near-normal (meridional) and near-tangential (longitudinal) satellite crossings of the OCB give rise to markedly different signatures. As the OCB is usually roughly L-shell aligned these near-normal and near-tangential crossings are meridional and longitudinal in nature. Under pulsed reconnection conditions, the model predicts that a meridional pass will measure a stepped ion signature, as reported by Newell and Meng (1991) and by Lockwood et al. (1993), whereas a longitudinal crossing will measure a sawtooth ion signature, as reported by Pinnock et al. (1995).

\subsection{Boundary definitions}

A common point of contention in current literature is the identification of the open-closed field line boundary (OCB). Here we define the polar cap as comprising of open fieldlines connected to the IMF, hence bounded by the OCB, but there are difficulties in locating the OCB using different types of data. For this reason there are many "working definitions" of the polar cap boundary, including the auroral oval bound- 
aries. Even here there is ambiguity as optical observations will give different boundaries, dependent on wavelength and intensity threshold, when compared with particle precipitation. There have been a number of studies linking the poleward auroral emission (PAE) boundary with the polar cap boundary (Elphinstone et al., 1990; Brittnacher et al., 1999) and while there are still many questions regarding the validity of this technique, the correlation between DMSP and UVI poleward auroral oval boundaries is generally good (Germany et al., 1997; Kauristie et al., 1999; Baker et al., 2000). On the dayside, separations between the OCB and the PAE tend to be small.

Sotirelis et al. (1998) have attempted to determine the shape of the OCB from just DMSP precipitating particle data, but had difficulties because the OCB shape is variable on much shorter temporal and spatial scales than the separation of the DMSP satellite passes in time and space, particularly under substorm conditions. Importantly, the lowlatitude boundary layer was taken as being inconsequential to their results, because it was assumed that it was of small and constant latitudinal extent - especially near magnetic noon. The responses of the convection reversal boundary (CRB) and the pattern of convection, in general, to IMF conditions have also been well studied (Cowley and Lockwood, 1992; Lester et al., 1995; McCrea et al., 2000). However, identification of the OCB from the CRB is difficult due to the variable offsets due to viscous-like momentum transfer to closed field lines and spatial gradients in ionospheric conductivities.

\section{Data sets}

\subsection{DMSP SSJ/4 spectrograms}

The Defense Meteorological Satellite Program (DMSP) operates several satellites, each equipped with identical lowand high-energy SSJ/4 precipitating particle detectors. These instruments measure electron and ion flux in logarithmically spaced energy bins from $30 \mathrm{eV}$ to $1 \mathrm{keV}$ and $1 \mathrm{keV}$ to $30 \mathrm{keV}$ - the redundancy at $1 \mathrm{keV}$ is used for cross-calibration of the low- and high-energy detectors. The satellites have a polar, sun-synchronous orbit at a height of $\sim 840 \mathrm{~km}$ with an orbital period of $\sim 102 \mathrm{~min}$.

The DMSP electron precipitation could be used to define the dayside OCB as the poleward edge of the full magnetosheath population. This assumes that magnetospheric electrons flow sufficiently rapidly across the magnetopause along newly-opened field lines into the magnetosheath, such that the offset caused by field line convection in the electrons' time-of-flight can be neglected. At almost the same location, the satellite should observe the equatorward edge of magnetosheath-like electrons that have flowed into the magnetosphere along the newly-opened field lines. Sometimes these signatures overlap, which may indicate an unknown mechanism is injecting sheath electrons onto closed LLBL field lines - placing the OCB at the poleward edge of the magnetospheric electrons. Alternatively, the magnetospheric
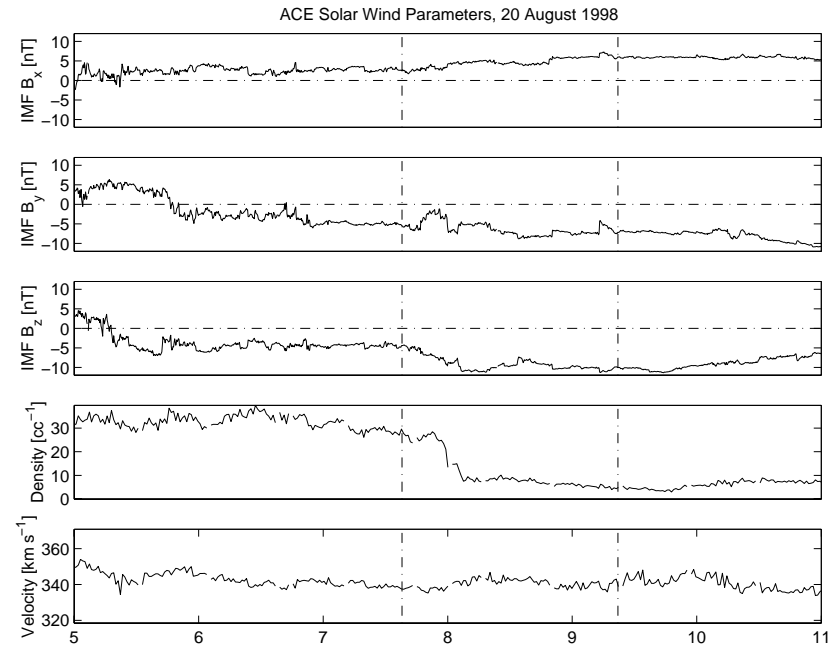

Fig. 1. IMF $B_{x}$ (top panel), $B_{y}$ (middle panel) and $B_{z}$ (bottom panel) data taken by the MAG instrument on board ACE for 20 August 1998. The vertical marks at 07:38 and 09:22 UT correspond to the lagged times of the DMSP cusp crossings at 08:54 UT and 10:38 UT, respectively.

electrons may have been prevented from escaping along open field-lines by a magnetic bottle or a potential drop or may have gradient-B and/or curl-B drifted from closed to open field lines - in which case the OCB is at the equatorward edge of the sheath electrons and the LLBL is open (Lockwood, 1997). In addition, a lack of substorm activity over the previous hours may have caused the energetic magnetospheric electron fluxes to have fallen to values below the instrument one-count level on some L-shells. Thus, their poleward edge is not always a reliable indicator of the OCB and is not used here. The satellite positions have been transformed to MLat/MLT coordinates using the Tsyganenko T89 magnetic field model with a standard reference height of $840 \mathrm{~km}$. Ion precipitation signatures have been shown to depend on the angle at which the satellite traverses the OCB (Lockwood and Davis, 1996), but we show that these signatures also depend on the convection velocity and reconnection rate.

This paper examines an event with the observed characteristic of a stepped ion dispersion followed by a sawtooth ion dispersion, and shows how these dispersions can be interpreted in terms of pulsed reconnection. The 20 August 1998 event was observed during consecutive cusp crossings by the DMSP F-14 craft at $\sim 08: 54$ UT and $\sim 10: 38$ UT, respectively.

\subsection{Interplanetary conditions}

The interplanetary conditions for the period under study (see Fig. 1) have been taken from the ACE satellite which is upstream of the Earth, along the Sun-Earth line at $\left(X_{\mathrm{GSE}}=\right.$ $248 R_{E}, Y_{\mathrm{GSE}}=12 R_{E}, Z_{\mathrm{GSE}}=24 R_{E}$, where $1 R_{E}$ is a mean Earth radius; $6370 \mathrm{~km}$ ). For this location we can be reasonably confident that most changes in IMF orientation measured by ACE will interact with the Earth. The time lag 

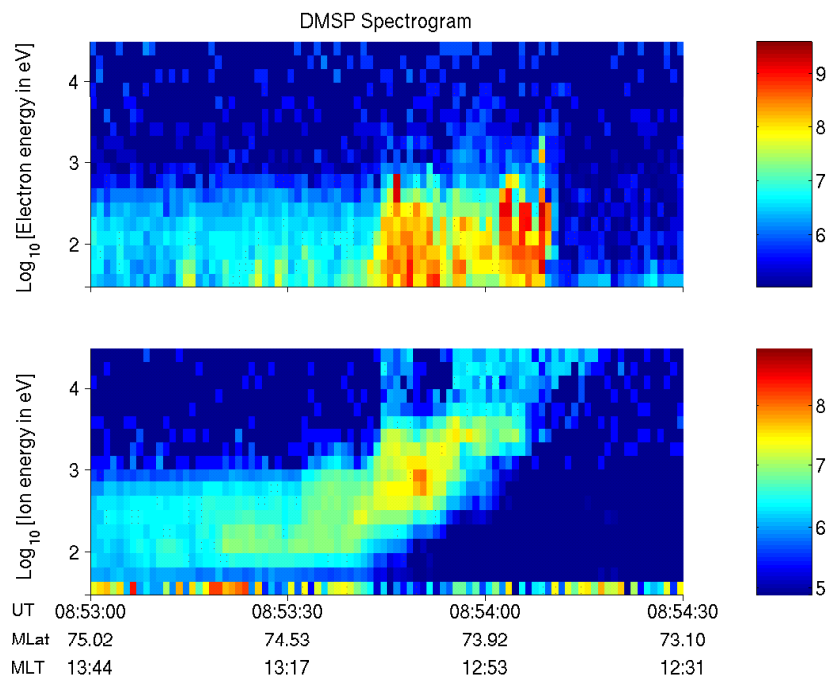

MLT $13: 44$

$13: 17$

$12: 53$

$12: 31$

Fig. 2. Energy-time electron and ion spectrogram observed by DMSP F-14 on 20 August 1998, with the cusp crossing from 08:53:36-08:54:09 UT.

from ACE to Earth for this event is estimated from the solar wind speed to be $76 \mathrm{~min}$. A southward turning of the IMF occurred at 05:18 UT. From about 04:30 UT to 08:00 UT the solar wind density was enhanced to an above average value of $n_{p} \sim 37 \mathrm{~cm}^{-3}$. For the remainder of the period the IMF was strongly southward $\left(B_{z}=-5\right.$ to $\left.-10 \mathrm{nT}\right)$, with strong and consistently negative IMF $B_{y}$. The solar wind speed was $\sim 345 \mathrm{~km} \mathrm{~s}^{-1}$; this remained fairly constant throughout the interval. The vertical dot-dash lines in Fig. 1 are at the lagged times of the two DMSP crossings studied in this paper. The IMF $B_{y}$ component is similar in the two cases $(\sim-5 \mathrm{nT})$, but the $B_{z}$ component is roughly twice as large for the second pass ( $\sim-8 \mathrm{nT}$ with $\sim-4 \mathrm{nT}$ for the first pass). Though the solar wind speed is roughly the same in the two cases, the concentration, $n_{p}$, is much lower $\left(\sim 5 \mathrm{~cm}^{-3}\right.$, compared to $\sim 30 \mathrm{~cm}^{-3}$ for the first pass).

The $A E$ index (data not shown) shows a period of substorm growth from roughly 06:36 UT. This corresponds to the arrival at Earth of the southward IMF seen at ACE at about 05:18 UT and thus estimated to arrive at the magnetosphere at about 06:34 UT, confirming the propagation lag of $76 \mathrm{~min}$.

\section{Results}

Energy-time spectrograms are presented in Figs. 2 and 3 for both electrons and ions, as observed by DMSP F-14. In these plots the differential energy flux is plotted both as a function of energy and time of observation.

On 20 August 1998, the DMSP F-14 satellite crossed the cusp just to the south of Longyearbyen, returning on the following orbit to similar (MLT, MLat) coordinates. In both these passes, energetic magnetospheric electron fluxes were too low to show any OCB signature, which is, therefore,

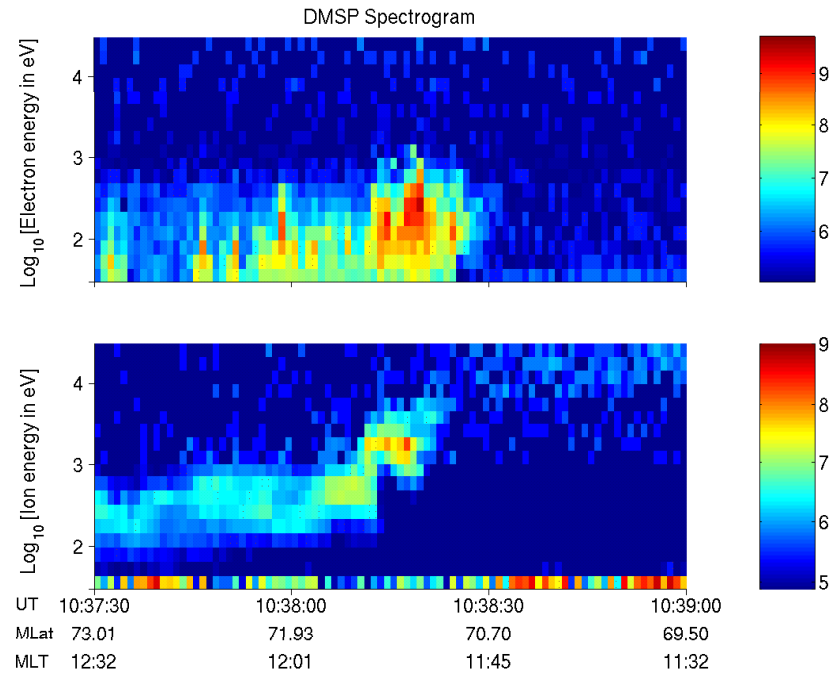

Fig. 3. Energy-time electron and ion spectrogram observed by DMSP F-14 on 20 August 1998, with the cusp crossing from 10:38:12-10:38:27 UT.

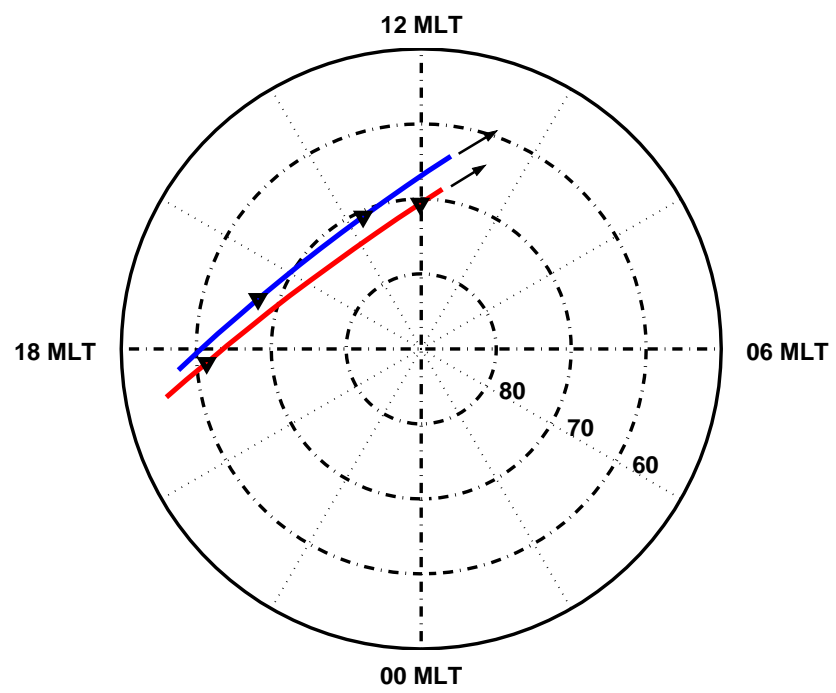

Fig. 4. Trajectories of DMSP F-14 on successive orbits, mapped in invariant latitude (MLAT) and magnetic local time (MLT). The blue track corresponds to the first satellite pass, the red track corresponds to the second satellite pass. The triangles mark the OCB boundaries as defined in precipitating particle data.

clearest in the sheath electron data. The orbit paths for these cusp passes are shown in Fig. 4. The cusp crossings were from 08:53:36-08:54:09 UT and 10:38:12-10:38:27 UT, respectively. The poleward emission boundary was observed by the UVI instrument on Polar (see Fig. 5) to move equatorward from about $74.3^{\circ}$ to $71.4^{\circ}$ MLat. The OCB determined from the equatorward edge of sheath electrons seen by F-14 was at $73.7^{\circ}$ MLat and $70.9^{\circ}$ MLat for the two passes, indicating an overlap of about $0.5^{\circ}$ in both cases. The electrons causing this overlap were not seen by the DMSP satellites (either because they were of too low a flux or because they were at energies above $30 \mathrm{keV}$ and thus out of the range of the 
UVI 19980820 0859:38 UT LEHL

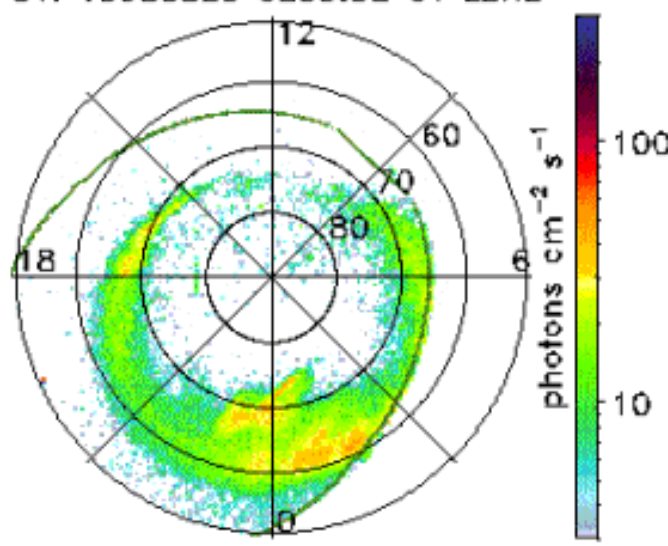

UVI 19980820 1039:37 UT LBHL

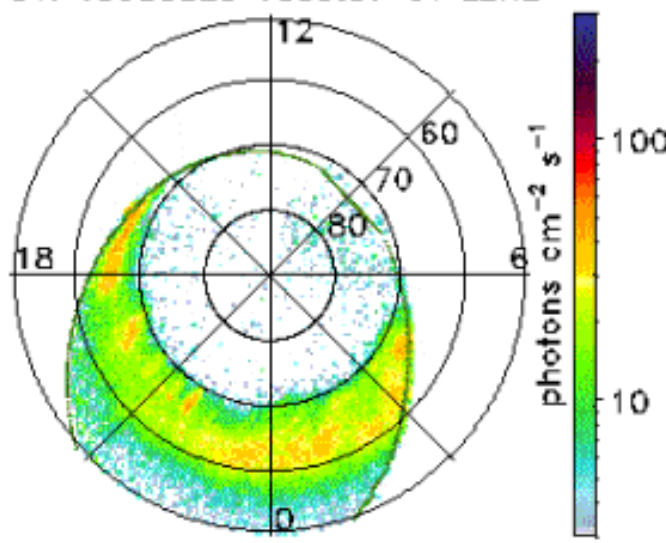

UVI 19980820 0911:54 UT LEHL

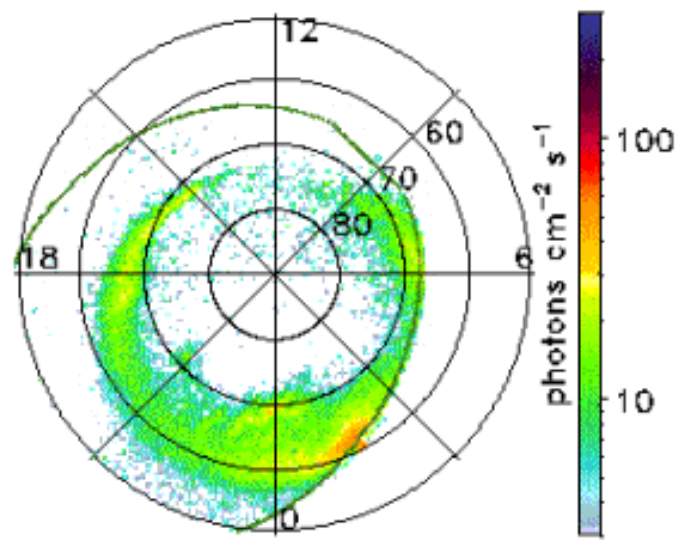

UVI 19980820 1048:49 UT LBHL

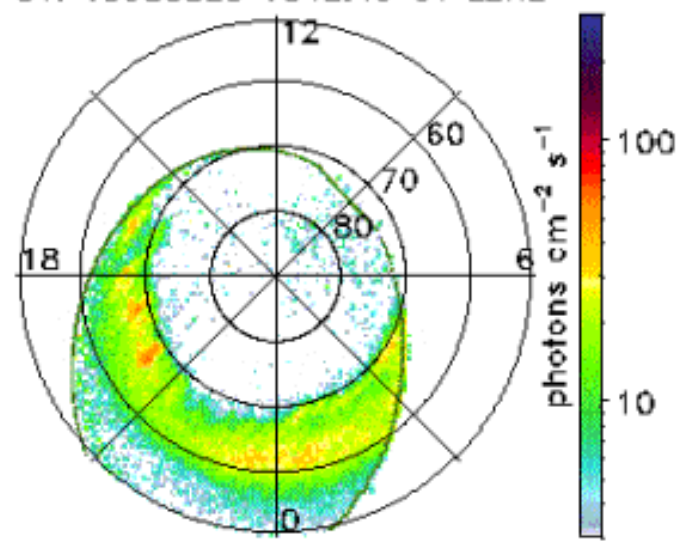

Fig. 5. Plots of the Polar UVI data at 08:59, 09:11, 10:39 and 10:48 UT, mapped in invariant latitude (MLat) and magnetic local time (MLT). The green line indicates the approximate field-of-view.

instrument). We can conclude that the LLBL, be it open or closed, was only about $0.5^{\circ}$ in latitudinal width. As the orbit precession is extremely slow, the satellite trajectory in each case is very similar. Both cusp crossings display structure in the ion dispersions, the first crossing exhibiting a stepped signature and the second crossing displaying a sawtooth signature. For the first pass upward steps are seen at 08:53:42 and 08:53:56 UT, and these are separated by upward dispersion ramps, a "stepped" signature. For the later pass an upward step is again observed at 10:38:13 UT. However, the dispersion ramp after this step is downward, giving a "sawtooth" appearance.

\section{Discussion}

We have presented two satellite passes with similar trajectories but with clearly different cusp ion dispersions. The IMF was southward in both cases. Following Lockwood and Davis (1996) we have searched for a change in the OCB orientation that could explain this. In the time between the two DMSP passes, the IMF $B_{y}$ has become slightly more negative, opening the possibility of an asymmetric expansion of the polar cap favouring the dusk sector. As the required change in orientation of the OCB is anti-clockwise, and a negative $B_{y}$ shift will tend to drag the merging gap dawnwards - hence clockwise - any such shift cannot provide the explanation. Though the field-of-view of UVI is limited at this time (see Fig. 5), inspection of UVI images shows no evidence of the OCB orientation change needed.

The observed density enhancement, when lagged to find the time of arrival at Earth, begins over $3 \mathrm{~h}$ before the first pass. The density then drops from about $30 \mathrm{~cm}^{-3}$, at 07:53 UT, to about $5 \mathrm{~cm}^{-3}$, at 08:07 UT (lagged times of 09:09 UT and 09:23 UT) - over an hour before the second pass. Any temporal effects with a causal link to the density drop will have dissipated by this time, so they will not affect the form of the signature observed. It can be seen, however, that the ion flux levels are somewhat lower in the second pass - consistent with this density change.

Structured cusp ion dispersions as a result of pulsed reconnection from an extended X-line have been modelled by Lockwood and Davis (1996), showing that different satellite trajectories can give rise to different ion dispersion signatures for the same reconnection conditions. The conditions for observing a stepped or sawtooth signature are dependent on the ratio of the satellite and boundary velocities (as this is treated 

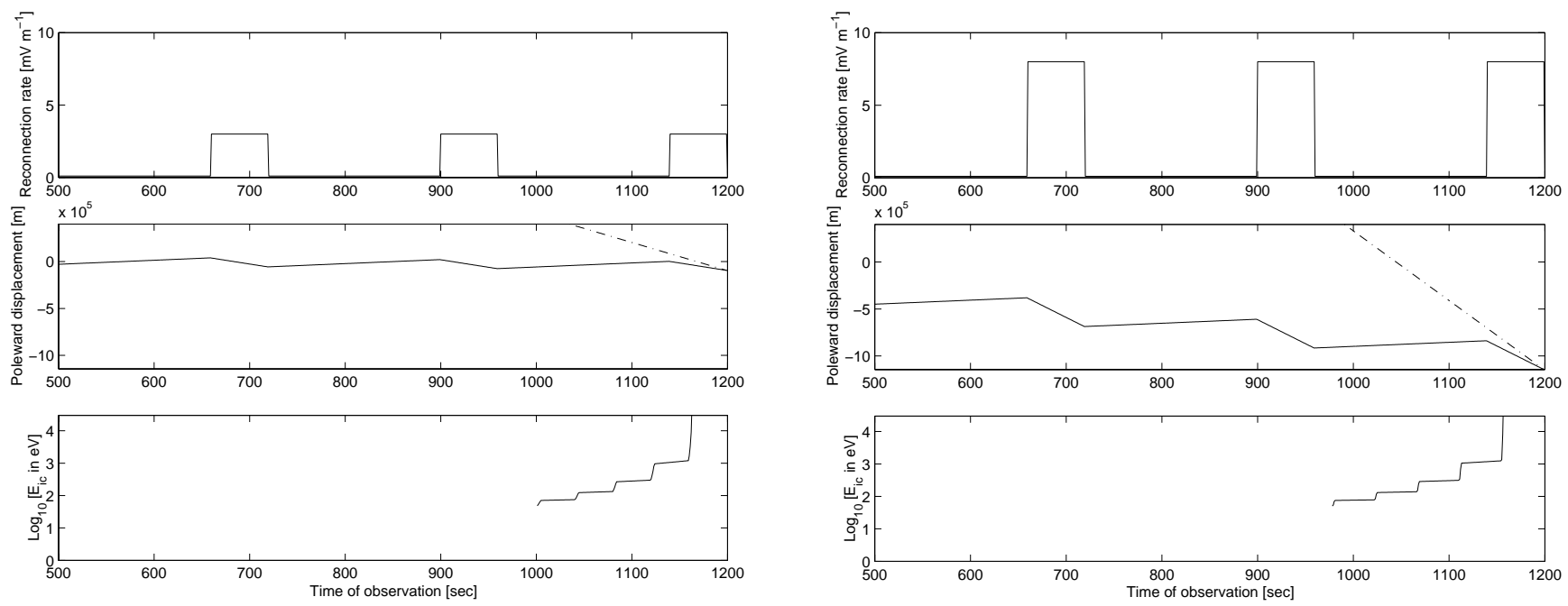

Fig. 6. A three-panel plot of: the input reconnection rate for the model under pulsed reconnection; relative positions of the satellite (dash-dot line) and OCB (solid line); and the modelled low-energy ion cutoff as observed within the energy range of the DMSP satellites. The model is using a 1-minute reconnection pulse within a 4-min cycle. Pulse reconnection rate, $\varepsilon_{p}=3 \mathrm{mV} \mathrm{m}^{-1}$; Background reconnection rate, $\varepsilon_{b}=0.01 \mathrm{mV} \mathrm{m}^{-1}$; Satellite velocity, $V_{S}=3 \mathrm{~km} \mathrm{~s}^{-1}$.
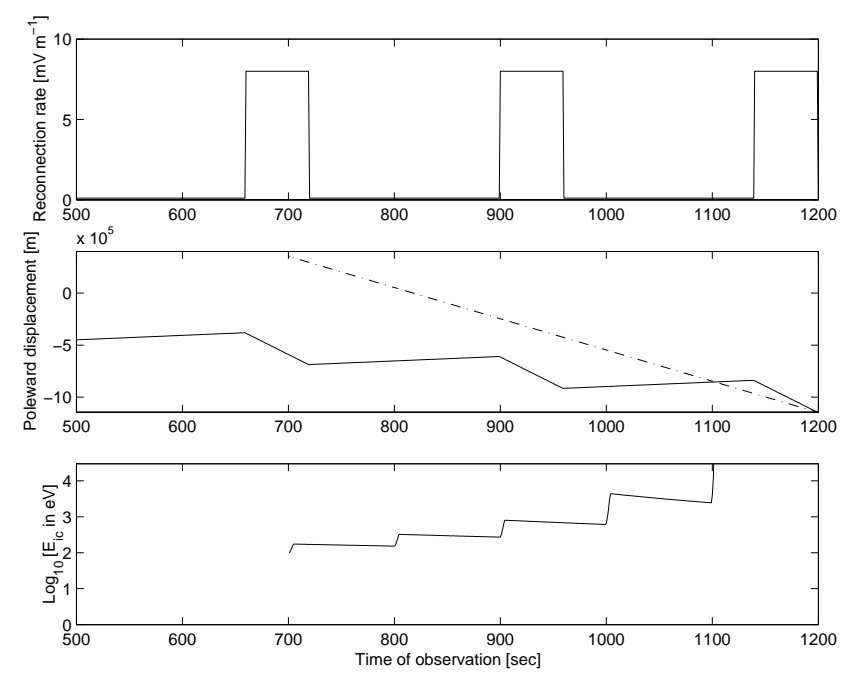

Fig. 7. Same as Fig. 6, but for $\varepsilon_{p}=8 \mathrm{mV} \mathrm{m}^{-1}$.

in 1-D, i.e. there is longitudinal symmetry, all velocities are considered normal to the merging gap) and are summarised here.

For a satellite moving poleward with the field lines (i.e. it is at rest in the de Hoffman-Teller frame), a steady-state dispersion will be observed, even though the reconnection rate is pulsed, because the satellite stays with the same newlyopened field line. If the satellite velocity exceeds the convection velocity significantly, a stepped signature will result. Similarly, if the convection velocity is much greater than the satellite velocity, then a sawtooth signature is observed, even

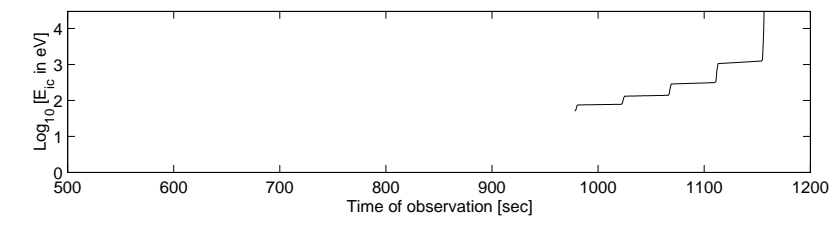

Fig. 8. Same as Fig. 7, but for $V_{s}=7 \mathrm{~km} \mathrm{~s}^{-1}$.

though the reconnection behaviour in space and time is the same. The plots presented here take equatorward erosion of the OCB into account and show how a variation in the reconnection rate can give both sawtooth and stepped signatures for identical satellite trajectories. The convection velocity is dependent on the average reconnection rate, as is the overall boundary drift.

If the satellite moves equatorward across the OCB (e.g. DMSP in the dayside Northern Hemisphere), then a stepped ion signature will be seen if the satellite boundary-normal velocity is greater than (or equal to) the boundary-normal speed of equatorward erosion. In this case the satellite will be moving from older flux tubes to more recently opened flux tubes. For the case of the satellite velocity being less than the erosion velocity, the relatively rapid erosion of the boundary means that the satellite can move onto a flux-tube that has been open for longer than the one it just left, hence a sawtooth signature is seen.

Lockwood and Davis (1996) considered the case of the same reconnection history and two different pass orientations relative to the OCB. Here we extend the analysis by considering different reconnection rate variations but the same pass orientation relative to the OCB. We have made calculations using a 1-D simulation of OCB dynamics under pulsed reconnection. The low-energy ion cutoff at the satellite is calculated as a function of observation time. The input reconnection rate behaviour can be pulsed and can include background reconnection between pulses. For the purposes of this paper convective flow is assumed to be constant during reconnection pulses (which have an assumed square-wave form). In other words, the pulses are inductively smoothed.

The OCB motion can be described as a sum of boundarynormal flow velocities. The boundary velocity, $V_{b}$, is governed by the reconnection rate, $\varepsilon$, and the convection velocity, $V_{c}$ (Lockwood and Smith, 1992):

$V_{b}=V_{c}-V^{\prime}=V_{c}-\left(\varepsilon / B_{i}\right) \cdot\left(d y^{\prime} / d y\right)$, 
where $V^{\prime}$ is the flow speed across the OCB in its own rest frame, $\left(d y^{\prime} / d y\right)$ is the mapping factor between the length of the reconnection $\mathrm{X}$-line and its ionospheric projection and $B_{i}$ is the ionospheric magnetic field strength.

Between the cusp crossings by the DMSP satellite the IMF $B_{z}$ component became more negative, suggesting a possible cause for the increased rate of reconnection. The average reconnection voltage is known to be dependent on the magnitude of $B_{z}$ (Reiff et al., 1981; Freeman et al., 1993; Fedder et al., 1991). This can occur because the X-line becomes longer or because the reconnection rate increases, or both.

The model predictions of Lockwood and Davis (1996) for the cusp ion dispersion signatures observed by satellites travelling meridionally and longitudinally, relative to the OCB can be reproduced in form by assuming a variation in reconnection rate (see Figs. 6 and 7). The key factor is the ratio of boundary-normal satellite velocity, $V_{s}$, to the equatorward boundary velocity, $V_{b}$. Changing the satellite trajectory will affect $V_{s}$, possibly affecting the observed cusp ion signature. However, if the rate of reconnection is varied, the conditions for the observation of these signatures can be met. The signatures calculated here are longer than is typically observed. Sawtooth signatures tend to be observed over greater periods, as they are generally associated with a skimming of the OCB. These have been observed to last for several hundred seconds (Pinnock et al., 1995). Stepped signatures, resulting from a high boundary-normal satellite velocity relative to the OCB, will be observed with a shorter duration (typically a few tens of seconds). The model assumes a stable system with regular reconnection pulses and a large inter-pulse period; also the one-dimension nature of the model means that the longitudinal extent of the X-line projection is not taken into account. The calculated signatures are intended to be illustrative and reproduce the form of the signature.

Figures 6,7 and 8 show the results of model calculations. In each case the top panels show the input square wave reconnection rate variation with pulses of amplitude $\varepsilon_{p}$, between which is a background rate of $\varepsilon_{b}$. The middle panels show the OCB latitude variation (solid line) and the satellite latitude (dot-dash line). The bottom panels show the predicted low-energy ion cutoff as a function of time as the satellite passes along the path shown in the middle panel. In each case, the poleward convection velocity, $V_{c}$, was set to a fixed (inductively smoothed) value of $500 \mathrm{~m} \mathrm{~s}^{-1}$ and the boundary motion was found by evaluating $V_{b}$ from Eq. (1). Note the scales used for each panel are common to all three figures. Figure 6 is for relatively small reconnection rate pulses and reveals a classic stepped signature as the satellite moves equatorward. Figure 7 uses the same reconnection rate variation, except the amplitude of the pulses, $\varepsilon_{p}$, is $(8 / 3)$ times larger. It can be seen that the stepped signature has become a sawtooth form. Figure 8 is for exactly the same reconnection rate behaviour as Fig. 7, but the spacecraft is moving along a path that has a much greater meridional component (as can be seen from the greater slope of the dot-dash line) and the gradient of the satellite position is once again greater than that of the OCB position during the reconnection pulse, i.e. $V_{s}>V_{b}$. It can be seen that this has returned the dispersion to a stepped form, the effect modelled by Lockwood and Davis (1996).

\section{Summary}

The form of cusp ion dispersion signatures was previously shown to be dependent on the trajectory of the observing satellite. Modelling has shown that reconnection rate can also determine the form of the cusp ion dispersion observed. Data from consecutive DMSP satellite passes on 20 August 1998 show both stepped and sawtooth signatures. Supporting data shows that an orientation change in the OCB is unlikely to explain the change in signature. The most likely cause of increased reconnection over this period is a $5 \mathrm{nT}$ increase in the magnitude of the already negative IMF $B_{z}$ that appears to have increased the amplitude of the reconnection pulses.

We conclude that the rate of reconnection must have increased - if the average reconnection voltage had increased by a lengthening of the $\mathrm{X}$-line alone, then the signature would not have changed form. The effect of lengthening the $\mathrm{X}$-line would be to increase the longitudinal extent of the reconnection pulses.

Acknowledgements. The authors would like to thank the ACE/MAG team for providing IMF data and G. Parks for supplying UVI data. The DMSP particle detectors were designed by Dave Hardy of AFRL, and data obtained from JHU/APL. We thank D. Hardy, F. Rich, and P. Newell for its use. This work was supported by PPARC (UK Particle Physics and Astronomy Research Council).

Topical Editor G. Chanteur thanks a referee for his help in evaluating this paper.

\section{References}

Baker, J. B., Clauer, C. R., Ridley, A. J., Papitashvili, V. O., Brittnacher, M. J., and Newell, P. T.: The nightside poleward boundary of the auroral oval as seen by DMSP and the Ultraviolet Imager, J. Geophys. Res., 105, 21 267-21 280, 2000.

Brittnacher, M., Fillingim, M., Parks, G., Germany G., and Spann, J.: Polar cap area and boundary motion during substorms, J. Geophys. Res., 104, 12 251-12 262, 1999.

Cowley, S. W. H. and Lockwood, M.: Excitation and decay of solar-wind driven flows in the magnetosphere-ionosphere system, Ann. Geophysicae, 10, 103-115, 1992.

Elphinstone, R. D., Jankowski, K., Murphree, J. S., and Cogger, L. L.: The configuration of the auroral distribution for interplanetary magnetic field $B_{z}$ northward, 1 . IMF $B_{x}$ and $B_{y}$ dependencies as observed by the Viking satellite, J. Geophys. Res., 95, 5791-5804, 1990.

Fedder, J. A., Mobarry, C. M., and Lyon, J. G.: Reconnection voltage as a function of IMF clock angle, Geophys. Res. Lett., 18, 1047-1050, 1991.

Freeman, M. P., Farrugia, C. J., Burlaga, L. F., Hairston, M. R., Greenspan, M. E., Ruohoniemi, J. M., and Lepping, R. P.: The interaction of a magnetic cloud with the Earth: Ionospheric convection in the northern and southern hemispheres for a wide 
range of quasi-steady IMF conditions, J. Geophys. Res., 98, 7633-7655, 1993.

Germany, G. A., Parks, G. K., Brittnacher, M. J., Cumnock, J., Lummerzheim, D., Spann, J. F., Chen, L., Richards, P. G., and Rich, F. J.: Remote determination of auroral energy characteristics during substorm activity, Geophys. Res. Lett., 24, 995-998, 1997.

Kauristie, K., Weygand, J., Pulkinnen, T. I., Murphree, J. S., and Newell, P. T.: Size of the auroral oval: UV ovals and precipitation boundaries compared, J. Geophys. Res, 104, 2321-2331, 1999.

Lester, M., Lockwood, M., Yeoman, T. K., Cowley, S. W. H., Lühr, J., Bunting, R., and Farrugia, C. J.: The response of ionospheric convection in the polar cap to substorm activity, Ann. Geophysicae, 13, 147-158, 1995.

Lockwood, M.: Identifying the open-closed field line boundary, in Proceedings of the NATO Advanced Study Institute on Polar Cap Boundary Phenomena, (Eds) Moen, J., Egeland, A., and Lockwood, M., Kluwer Academic Press, Dordrecht, The Netherlands, 73-90, 1997.

Lockwood, M., Denig, W. F., Farmer, A. D., Davda, V. S., Cowley, S. W. H., and Lühr, H.: Ionospheric signatures of pulsed reconnection at the Earth's magnetopause, Nature, 361, 424-427, 1993.

Lockwood, M. and Smith, M. F.: The variation of reconnection rate at the dayside magnetopause and cusp ion precipitation, J. Geophys. Res., 97, 14 841-14 847, 1992.

Lockwood, M. and Davis, C. J.: On the longitudinal extent of magnetopause reconnection, Ann. Geophysicae, 14, 865-878, 1996.

McCrea, I. W., Lockwood, M., Moen, J., Pitout, F., Eglitis, P., Aylward, A. D., Cerisier, J.-C., Thorolfssen, A., and Milan, S. E.: ESR and EISCAT observations of the response of the cusp and cleft to IMF orientation changes, Ann. Geophysicae, 18, 10091026, 2000.

Newell, P. T. and Meng, C.-I.: Ion acceleration at the equatorward edge of the cusp: low-altitude observations of patchy merging, Geophys. Res. Lett., 18, 1829-1832, 1991.

Pinnock, M., Rodger, A. S., Dudeney, J. R., Baker, K.-B., Newell, P. T., Greenwald, R. A., and Greenspan, M. E.: High spatial and temporal resolution observations of the ionospheric cusp, Ann. Geophysicae, 13, 919-925, 1995.

Reiff, P. H., Spiro, R. W., and Hill, T. W.: Dependence of polar cap potential drop on interplanetary parameters, J. Geophys. Res., 86, 7639-7648, 1981.

Sotirelis, T., Newell, P. T., and Meng, C.-I.: Shape of the openclosed boundary of the polar cap as determined from observations of precipitating particles by up to four DMSP satellites, J. Geophys. Res., 103, 399-406, 1998. 